\title{
Early Movement Monitoring Assessment in Greek Full-Term and Preterm Infants with Normal and Low Birth Weight: Pilot Study
}

\author{
Niki Karageorgi, Sofia Charitou, Katerina Asonitou, Dimitra Koutsouki \\ Laboratory of Adapted Physical Activity, Developmental and Physical Disabilities, School of Physical Education and Sport Science, \\ National \& Kapodistrian University of Athens, Athens, Greece \\ Email: nkarageorgi@phed.uoa.gr
}

How to cite this paper: Karageorgi, N., Charitou, S., Asonitou, K., \& Koutsouki, D. (2021). Early Movement Monitoring Assessment in Greek Full-Term and Preterm Infants with Normal and Low Birth Weight: Pilot Study. Advances in Physical Education, 11, 284-293.

https://doi.org/10.4236/ape.2021.112024

Received: April 7, 2021

Accepted: May 25, 2021

Published: May 28, 2021

Copyright $\odot 2021$ by author(s) and Scientific Research Publishing Inc. This work is licensed under the Creative Commons Attribution International License (CC BY 4.0).

http://creativecommons.org/licenses/by/4.0/

(c) (i) Open Access

\begin{abstract}
Objective: To investigate if Early Movement Monitoring Assessment (EMMA) is a sesitive tool to the developmental changes across time, in frequency of total movements in Greek full term and preterm infants with normal and low birth weight. Methods: EMMA and Alberta Infant Motor Scale (AIMS) were performed on Greek infants, who were born full-term and pre-term (age corrected), with normal or low birth weight. Specifically, 11 full-term infants, 4 preterm infants with normal birth weight $(>2500 \mathrm{gr})$ and 5 preterm infants with low birth weight $(<2500)$ were assessed with EMMA for 4 - 6 sessions to meet the purpose of the study. AIMS was used as criterion measurement for validity reasons. Descriptive statistics were conducted (Mean, SD) and correlation analysis was also applied between EMMA and AIMS age groups. Results: Significant correlation between EMMA and AIMS was found. Results draw a positive slope for each group, indicating that EMMA was sensitive to change in motor skills across time. Conclusion: In Greece there are the preliminary evidences to consider that EMMA is a valid tool which can be used to assess motor development easily, quickly and frequently for full-term and preterm infants with normal or low birth weight.
\end{abstract}

\section{Keywords}

Early Movement Monitoring Assessment, Motor Development, Infants, Monitoring, Full-Term Infants, Pre-Term Infants

\section{Introduction}

Neuronal development is continuously changing during the formative years of infancy and toddlerhood (Hadders-Algra, 2001). Movement in the first years of 
life is considered to strengthen brain development and cognitive aspects (MacVarish, Ellie, \& Pam, 2014). Movement skills development in infancy is the most rapid than any other time during life (Gabbard, 2012). According to World Health Organization (WHO), premature births occur at $10.6 \%$ worldwide (WHO, 2012). It is a fact that premature infants are at risk of developing neuro-developmental disorders due to the immature brain development, which is caused by many factors during the prenatal, perinatal, and postnatal period (Arpino et al., 2010; Volpe, 2009). More and more preterm infants survive because of obstetrical technology progress. However, children born preterm confront a variety of problems compared to their peers, regarding physical disability, cognitive, executive and motor functions, developmental coordination disorders, learning etc. as signs of deficits at the infancy (Marlow, Hennessy, Bracewell, \& Wolke, 2007; Orton, Spittle, Doyle, Aderson, \& Boyd, 2009; Stephens \& Vohr, 2009). Early intervention impels competencies, reduces developmental delays, and strengthens families' adaptation (Blauw-Hospers \& Hadders-Algra, 2005). Motor development is essential during the procedure of growth (Pipper \& Darrah, 1994), for example, crawling and walking enable the development of cognitive and social skills (Campos et al., 2000). Progress monitoring of motor function in infants can report documentation of established skills in the right time and the kind of intervention which is substantial for developing the following key skills (Leitschuh, Harring, \& Dunn, 2014).

As motor deficits are usually observed in preterm infants, monitoring early intervention programs should be completed with the appropriate available tools that are sensitive to evaluate the effectiveness of the intervention in the infants' motor development (Van Hus et al., 2013). There are plenty of assessment tools for infancy and toddlerhood testing motor development. Most of them use comprehensive developmental milestones. There is an essential merit in those, standardized, norm referred tests that are used traditionally. They manage to present where the subject stands regarding to its peers. Another method to report motor function is the "progress monitoring", which is a vital element of assessing. It is used widely in many domains such as education, special education, and many others. It is composed by short time consuming tasks, which reflect the desired or that specific key element, which would be useful to be observed, in order to plan a more detailed intervention program, or to correct the one that is already followed (Stecker, Lembke, \& Foegen, 2008). Progress monitoring also keeps the specialists or even parents alert when subjects' performance lucks to follow progress at acceptable rates. Progress monitoring tests are also quite short in time as they are completed in few minutes in contrast to traditional tools of assessment and finally as they are performed frequently, they do manage to track the growth.

Monitoring tools for motor development in infancy could be a useful knowledge to a professional as it can be ascertained in a few minutes if the infants' growth progress is at the expected level at a specific time of assessment or intervention is required. As preterm infants often luck to follow the track of growth 
in the same conditions that full term infants do, Early Monitoring Movement Assessment could be used to investigate that the growth is on track for those infants.

Summarizing, the EMMA is a progress monitoring tool which indicates the track that infants' motor development follows continuously, in order to note the earliest warnings or delays, quickly and easily, and finally check or improve an intervention program. The purpose of this pilot study was to investigate EMMA's sensitivity to developmental change over time among Greek infants who were born full-term and pre-term (age corrected) with normal or low birth weight.

\section{Method}

\subsection{Participants}

The subjects of this study were 20 infants ranged from 6 to 36 months old, who were accessed every 3 weeks for 4 - 6 sessions till the end of the research. These participants were divided by their gestational age and birth weight at 3 groups. Group 1 included 11 full-term ( $\mathrm{M}=39$ weeks, $\mathrm{SD}=0.52 / \mathrm{M}=3212 \mathrm{gr}, \mathrm{SD}=290)$ infants, group 2 included 4 pre-term with normal birth weight $(<37$ weeks, $>2500 \mathrm{gr})(\mathrm{M}=36.5$ weeks $\mathrm{SD}=1.15 / \mathrm{M}=2762 \mathrm{gr}, \mathrm{SD}=69.4)$ and group 3 included 5 preterm infants with low birth weight ( $<37$ weeks, $<2500 \mathrm{gr})(\mathrm{M}=$ 31.1 weeks, $\mathrm{SD}=1.6 / \mathrm{M}=1974 \mathrm{gr}, \mathrm{SD}=162)$, corrected age for the preterm infants (Table 1). Infants diagnosed with cerebral palsy or other impairments were not included in the research. The sample derived from collaboration with individual parents.

\subsection{Measures}

Early Movement Monitoring Assessment (EMMA)

The Early Movement Monitoring Assessment (EMMA) is a progress monitoring tool, which is sensitive to change over time in motor skills, valid, reliable, standardized and very quick (Leitschuh, Harring, \& Dunn, 2014). The EMMA is based on both ecological (Bronfendbrenner, 1977) and neuro-maturational theories (Gesell, 1925, 1945; McGraw, 1945) of motor development. The purpose of EMMA is to identify potential motor delays at a very fist level to note the necessity

Table 1. Demographic characteristics of the sample by groups' averages.

\begin{tabular}{|c|c|c|c|c|c|c|}
\hline Groups/Means & $\mathrm{N}$ & $\begin{array}{l}\text { Gestational } \\
\text { age (weeks) }\end{array}$ & $\begin{array}{l}\text { Birth weight } \\
\text { (grams) }\end{array}$ & $\begin{array}{l}\text { Age at first } \\
\text { testing time } \\
\text { (months) }\end{array}$ & Gender & $\begin{array}{l}\text { Sessions of } \\
\text { monitoring }\end{array}$ \\
\hline Full-term infants & 11 & $\begin{array}{l}M=39 w \\
S D=0.52\end{array}$ & $\begin{array}{c}3212 \mathrm{gr} \\
\mathrm{SD}=290\end{array}$ & $\begin{array}{c}14.8 \mathrm{~m} \\
\mathrm{Sd}=3.4\end{array}$ & $\begin{array}{c}\text { Male: } 6 \\
\text { Female: } 1\end{array}$ & 4 \\
\hline Preterm infants (NBW) & 4 & $\begin{array}{c}M=36.5 \mathrm{w} \\
\mathrm{SD}=1.15\end{array}$ & $\begin{array}{c}2762 \mathrm{gr} \\
\mathrm{SD}=69.4\end{array}$ & $\begin{array}{c}9.5 \mathrm{~m} \\
\mathrm{SD}=3.46\end{array}$ & $\begin{array}{c}\text { Male: } 3 \\
\text { Female: } 1\end{array}$ & 6 \\
\hline Preterm infants (LBW) & 5 & $\begin{array}{c}M=31.1 \mathrm{w} \\
\mathrm{SD}=1.6\end{array}$ & $\begin{array}{c}1974 \mathrm{gr} \\
\mathrm{SD}=162\end{array}$ & $\begin{array}{c}13.7 \mathrm{~m} \\
\mathrm{SD}=3.66\end{array}$ & $\begin{array}{c}\text { Male: } 2 \\
\text { Female: } 3\end{array}$ & 6 \\
\hline
\end{tabular}


of early intervention programs. The EMMA assesses in two minutes period using a 2-minute standardized protocol during play with specific toys, every 3 or 4 weeks. Specifically, EMMA records the frequency of transitioning in positions and locomotion skill as these are evidence behaviors in infancy. Transition in Position behavior refers to: prone, supine, side lie, sit, pull to kneel, kneel, pull to stand, stand, and stoop. Locomotion skill behavior refers to: crawl, crawl over, scoot, cruse flat, cruise up/down, walk, and run. EMMAs' toys are two balls, one toy car and one pushcart at standardized sizes suitable for infants and toddlers. The record form consists of pictures on which the observer marks the frequency of each movement. Total score is the results of both numbers of frequency in transitioning in positions and locomotion skill. It is referred to 0 - 36-month-old infants. Leitschuh and colleagues (2014), reported interrater reliability that exceeded 0.95. Criterion related validity with Mobility Screening Subtest of TIME (Miller \& Roid, 1994) were significant $\mathrm{r}=0.79, p=0.01$.

Alberta Infant Motor Scale (AIMS)

The purpose of The Alberta Infant Motor Scale (AIMS) (Pipper \& Darrah, 1994 ) is to evaluate motor development competence in infants aged up 18 months old. The AIMS include 58 items in four positions: prone ( 21 items), supine (9 items), sitting (12 items) and standing (16 items). The researcher marks any observed item at all four positions. Total score is summed up by the raw sub sores for each position. Then the total score is transformed to percentile ranks as this tool uses norms. AIMS is a global commonly used assessment in motor development in infancy. Test retest reliability is ranging from 0.85 to 0.99 (Charitou, Simitsopoulou, Kontogianni, Skordilis, \& Koutsouki, 2007; Pekçetin, Ak1, Üstünyurt, \&Kayıhan, 2016; Pipper \& Darrah, 1994; Syrengelas et al., 2010).

\subsection{Procedure}

Parents were informed about the purpose of the study, the data protection protocol as well as the right to desist any time from the research. Then, the parents signed a letter of consent. Every session took place at each infant's home in a quiet room that there were no other individuals apart from the researcher and the familiar caregiver. At the start of every session the researcher spent some time in the room in order the infant felt comfort with her presence. After ensuring that, the assessment was begun. The familiar caregiver sat close to the infant and placed the child either in a supine position or in an independent sit depending on infants' age. Toys were placed close to the infants to reach them. The researcher videotaped the procedure from $1.5 \mathrm{~m}$ distance approximately, for 2 minutes, for each child, every 3 weeks for 4 - 6 sessions. After calling infants' name the caregiver pointed the toys and asked to show how he/she plays with the toy that the infant is closed to or already holding. This prompt was repeated every 15 seconds after researchers' signal as it is described in EMMAS' research protocol (Leitschuh, Harring, \& Dunn, 2014). After recording the frequency of any coded movements, they were written down on the Record Form by one hatch mark for every movement that last 2 second and more. Then the child 
continued to play freely to observe AIMS coded behavior for each of its four positions as AIMS was used as a criterion measure tool. The observed behavior was also recorded on AIMS record booklet.

\subsection{Statistical Analysis}

Descriptive statistics were conducted (Mean, SD). Correlation analysis also applied between EMMA and AIMS age groups, as AIMS was our criterion measurement for validity reasons. Then, in order to investigate the rate of change in motor skills over time for the EMMA, data were recorded for each session as the total movements which combined transition in position and locomotor skills. Statistical analysis of the data was performed using SPSS statistics (version 25.0) for Windows. A significance $p$-value was set at $<0.05$.

\section{Results}

\subsection{Correlation Analyses between EMMA and AIMS}

The Alberta Infant Motor Scale (AIMS) (Pipper \& Darrah, 1994) in our study was employed as a golden standard tool. In a subgroup of the study participants that were suitable according to their age at testing time AIMS was administered. In our study, the sample aged between 6 - 36 months old. The researcher was trained in the administration of AIMS from an expert researcher of Adapted Physical Activity Developmental and Physical Disabilities of University of Athens Laboratory. The researcher videotaped and assessed 10 sessions. Then both the assessments and the tapes were viewed by the expert. The expert confirmed the AIMS had been administrated correctly at each session. Pearson correlation employed between researchers, the interrater reliability was 0.95 . Then, correlation analysis was conducted between the AIMS and the EMMA at the age groups 6 - 12 months and 12.1 - 18 months old for preterm infants, as this group had the suitable age for the AIMS assessment. The trend indicated strong correlation between AIMS and EMMA for both age groups $(\mathrm{r}=0.961, p=0.03)$ and $(\mathrm{r}=-0.985, p=0.05)$ respectively (Table 2$)$.

\subsection{Full-Term Infants (FT)}

Amongst the 11 full-term infants that participated in this study, missing data were occurred as they referred in Table 3. No missing data were observed for the first two sessions. One infant was not recorded at the third session and two at the fourth. Along, four sessions are a basic pattern to investigate developmental trajectories in this kind of research. It was observed that the total rates of movements grew over time for the full-term infants' group. The trend line at Figure 1 shows that there is a positive slope for full-terms infants, indicating that EMMA did picked up the fact that infants grew in their motor skills across time.

\subsection{Pre-Term Infants with Normal Birth Weight (PT-NBW)}

To investigate if EMMA is sensitive in developmental trajectories over time for 
Table 2. Correlation analyses between EMMA and AIMS.

\begin{tabular}{|c|c|c|c|c|c|}
\hline & & \multirow{2}{*}{$\begin{array}{c}\text { AIMS } \\
6-12 \text { months }\end{array}$} & \multirow{2}{*}{$\begin{array}{c}\text { EMMA } \\
6-12 \text { months }\end{array}$} & \multirow{2}{*}{$\begin{array}{c}\text { AIMS } \\
12-18 \text { months } 12\end{array}$} & \multirow{2}{*}{$\begin{array}{l}\text { EMMA } \\
2-18 \text { months }\end{array}$} \\
\hline & & & & & \\
\hline \multirow{3}{*}{$\begin{array}{c}\text { AIMS } \\
6-12 \text { months }\end{array}$} & Pearson correlation & 1 & $0.961^{\star}$ & & \\
\hline & Sig. (2-tailed) & & 0.39 & & \\
\hline & $\mathrm{N}$ & 4 & 4 & & \\
\hline \multirow{3}{*}{$\begin{array}{c}\text { EMMA } \\
6-12 \text { months }\end{array}$} & Pearson correlation & $0.961^{*}$ & 1 & & \\
\hline & Sig. (2-tailed) & 0.39 & & & \\
\hline & $\mathrm{N}$ & 4 & 4 & & \\
\hline \multirow{3}{*}{$\begin{array}{c}\text { AIMS } \\
12-18 \text { months }\end{array}$} & Pearson correlation & & & 1 & $-0.985^{*}$ \\
\hline & Sig. (2-tailed) & & & & 0.015 \\
\hline & $\mathrm{N}$ & & & 4 & 4 \\
\hline \multirow{3}{*}{$\begin{array}{c}\text { EMMA } \\
12-18 \text { months }\end{array}$} & Pearson correlation & & & $-0.985^{*}$ & 1 \\
\hline & Sig. (2-tailed) & & & 0.015 & \\
\hline & $\mathrm{N}$ & & & 4 & 4 \\
\hline
\end{tabular}

${ }^{*}$ Correlation is significant at the 0.05 level.

Table 3. Missing data across the six sessions for full-term infants.

\begin{tabular}{ccccc}
\hline & Session 1 & Session 2 & Session 3 & Session 4 \\
\hline Total number of participants & 11 & 11 & 10 & 9 \\
Missing Data & 0 & 0 & 1 & 2 \\
\hline
\end{tabular}

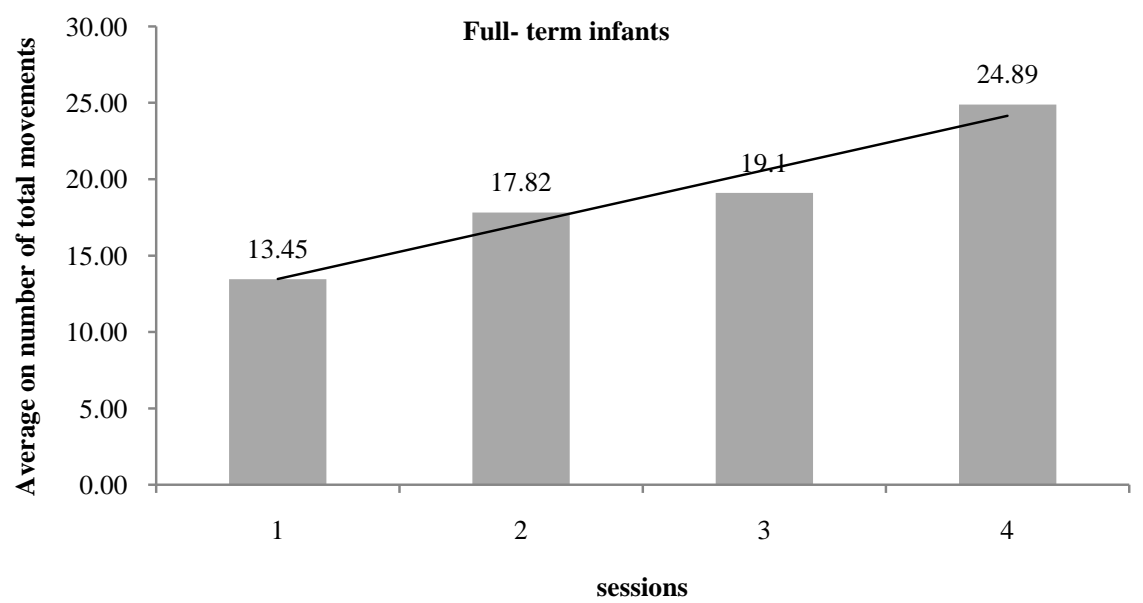

Figure 1. Average number of total movements of full-term infants over four sessions (3 weeks intervals).

preterm infants, four pre-term infants with normal birth weight participated in this study. No missing data were occurred in this group of infants. The results show that EMMA was sensitive to monitor motor skills during development in this sample of preterm infants with birth weight over 2500 gr (Figure 2). Figure 2 shows that there is a positive slope indicating that EMMA was sensitive to change in skill over time.

\subsection{Pre-Term Infants with Low Birth Weight (PT-LBW)}

In our sample there were 5 infants with birth weight less than 2500 grams. Missing 


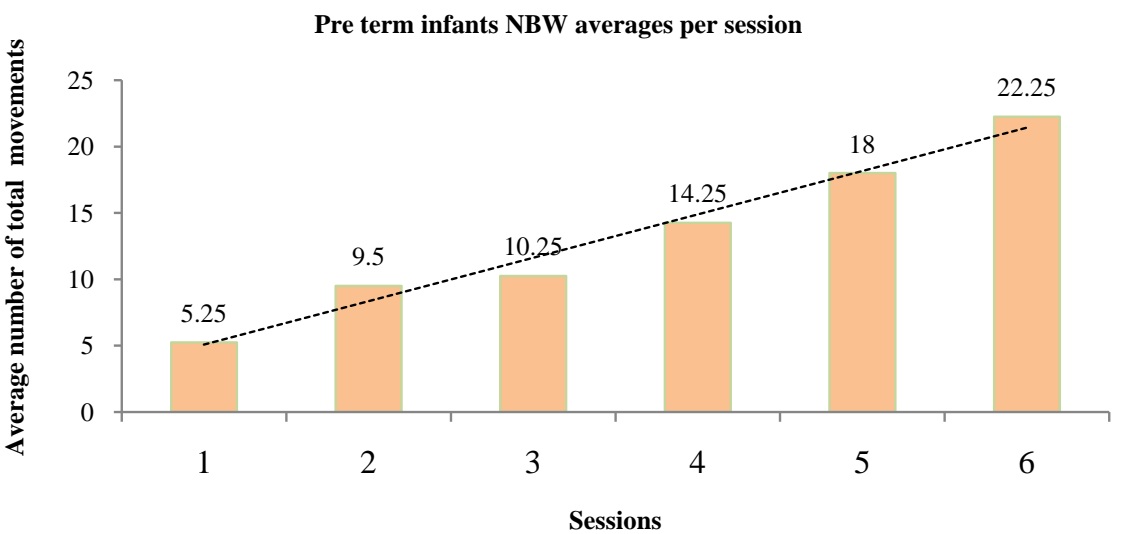

Figure 2. Total movements of preterm infants with normal birth weight over six sessions (3 weeks intervals).

Data occurred only for the last two sessions as Table 4 refers. Across the study period, preterm infants with low birth weight improved their total number of movements as the trend line shows a positive slope in Figure 3. Despite the slower rate, the positive slope shows that preterm infants with low birth weight did increase the skills performed.

\section{Discussion}

Traditional tools of motor development produce standard scores which indicate a specific status of movement at a specific time compared with norms. These tools luck to present the slope or rate of growth over time when considering monthly intervention programs (Greenwood, Carta, \& McConnell, 2011). Monitoring tools record skills in frequent intervals such as weeks. As a result, they can create trend lines across short sessions. Fundamental motor skills could be used as indicators of development instead of comprehensive developmental milestones (Leitschuh, Harring, \& Dunn, 2014). This is quite useful as when a lag is observed, intervention programs should be reconsidered. This pilot study was designed to examine if EMMA is sensitive to track the rate of total movements over time in Greek infants who were full-term, preterm (age corrected) with normal birth weight and preterm (age corrected) with low birth weight.

Initially, AIMS was used for validity reasons. The results showed that there was a strong correlation between AIMS and EMMA, indicating that despite the small size of the sample, this trend allows us to consider that EMMA is an adequate and valid tool, which could be used to access motor development in infants and toddlers. It should be noted that in our sample, we used data only from the two preterm groups, as only those infants had the appropriate age for the AIMS. This is a limitation of our study but still it is important to underline that there is a trend to indicate that EMMA could also be used for preterm infants. Leitschuh et al. (2014) used another comprehensive assessment, the Mobility Screening Subtest of the Toddler and Infant Motor Evaluation (TIME) (Miller \& Roid, 1994) as criterion tool for validity reasons and found significant correlation $(\mathrm{r}=0.79, p=0.01)$, too. 
Table 4. Missing data across the six sessions for preterm with low birth weight infants.

\begin{tabular}{ccccccc}
\hline & Session 1 & Session 2 & Session 3 & Session 4 & Session 5 & Session 6 \\
\hline Total number of participants & 5 & 5 & 5 & 5 & 4 & 4 \\
Missing Data & 0 & 0 & 0 & 0 & 1 & 1 \\
\hline
\end{tabular}

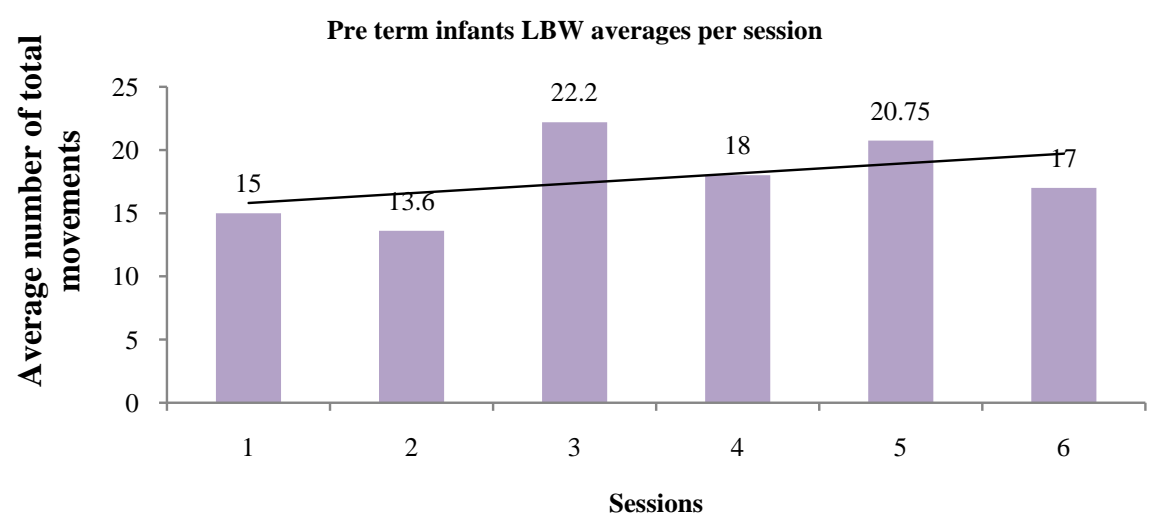

Figure 3. Total movements of preterm infants with low birth weight over six sessions (3 weeks intervals).

Results indicated that EMMA was sensitive to changes in the rate of total movements over time in Greek infants who were full-term, preterm (age corrected) with normal birth weight and preterm (age corrected) with low birth weight. Total movement rate grew across time for every group. The trend lines showed positive slopes for every group, which underlies that EMMA detected the fact that children grew in their motor skills across time. Leitschuh et al. (2014) found that EMMA was sensitive to follow the track of the changes over time in frequency of movement skills in full term infants. In their article they refer an unpublished pilot study using EMMA in premature infants. The researchers mentioned: "Those infants weighting less than $1400 \mathrm{gr}$, grew their motor skills despite a delay at the start". No other published study had used EMMA for preterm infants. In Greece we have the preliminary evidences to consider that EMMA is a valid tool which can be used to assess motor development easily, quickly and frequently for full-term and preterm infants with normal or low birth weight.

EMMA is a monitoring tool which is useful for professionals in order to investigate if the development is on track, to detect for developmental lags using key indicators of development instead of milestones, to review their interventional programs and make improvements, to produce a trend "profile" line across time on a monthly basis as motor development is changing very fast in early childhood. EMMA has social and practical applications for both specialist and therapists, as it is already mentioned, but also for parents. Up today, EMMA has not been used by parents although it is quick, easy and its toys are affordable to purchase and easy to procure. Especially, parents of preterm infants are of lasting concern regarding their children development. EMMA could be useful 
for them in order to note their infants track and take consider asking for professionals' intervention.

\section{Acknowledgements}

We would like to thank all the parents and infants that contributed to conduct this study.

\section{Conflicts of Interest}

The authors declare no conflicts of interest regarding the publication of this paper.

\section{References}

Arpino, C., Coompagnone, E., Montanaro, M. I., Cacciatore, D., De luca, A., Cerulli, A. et al. (2010). Preterm Birth and Neurodevelopental Outcome: A Review. Child's Nervous System, 26, 1139-1149. https://doi.org/10.1007/s00381-010-1125-y

Blauw-Hospers, C., \& Hadders-Algra, M. (2005). A Systematic Review of the Effects of Early Intervention on Motor Development. Developmental Medicine and Child Neurology, 47, 421-432.

Bronfenbrenner, U. (1997). Toward an Exerimental Ecology of Human Development. American Phychologist, 32, 513-531. https://doi.org/10.1037/0003-066X.32.7.513

Campos, J. J., Anderson, D. I., Barbu-Roth, M. A., Hubbard, E. M., Hertenstein, M. J., \& Witherington, D. (2000). Travel Broadens the Mind. Infancy, 1, 149-219. https://doi.org/10.1207/S15327078IN0102 1

Charitou, S., Simitsopoulou, A., Kontogianni, V., Skordilis, E., K., \& Koutsouki, D. (2007). Application of the Alberta Infant Motor Scale in Greece: A Preliminary Study. Inquiries in Sport \& Physical Education, 5, 273-282.

Gabbard, C. (2012). Lifelong Motor Development (6th ed.). San Francisco, CA: Pearson.

Gesell, A. (1925). The Mental Growth of the Preschool Child. New York: Macmillan.

Gesell, A. (1945). The Embryology of Behavior. New York: Harper Brothers.

Greenwood, C. R., Carta, J. J., \& McConnell, S. (2011). Advances in Measurement for Universal Screening and Individual Progress Monitoring of Young Children. Journal of Early Intervention, 33, 254-267. https://doi.org/10.1177/1053815111428467

Hadders-Algra, M. (2001) Evaluation of Motor Function in Young Infants by Means of the Assessment of General Movements: A Review. Pediatric Physical Therapy, 13, 27-36. https://doi.org/10.1097/00001577-200104000-00005

Leitschuh, C. A., Harring, J. R., \& Dunn, W. (2014). A Monitoring Tool of Infant and Toddler Movement Skills. Journal of Early Intervention, 36, 18-36.

https://doi.org/10.1177/1053815114555574

MacVarish, J., Ellie, L., \& Pam L. (2014). The "First Three Years" Movement and the Infant Brain: A Review of Critiques. Sociology Compass, 8, 792-804. https://doi.org/10.1111/soc4.12183

Marlow, N., Hennessy, E. M., Bracewell, M. A., \& Wolke, D. (2007). Motor and Executive Function at 6 Years of Age after Extremely Preterm Birth. Pediatrics, 120, 793-804. https://doi.org/10.1542/peds.2007-0440

McGraw, M. B. (1945). The Neuromuscular Maturation of the Human Infant. New York: Columbia University Press. 
Miller, L. J., \& Roid, G. H. (1994). The TIME Toddler and Infant Motor Evaluation: A Standardized Assessment. Tucson, AZ: Therapy Skill Builders. https://doi.org/10.1037/t15158-000

Orton, J., Spittle, A., Doyle, L., Anderson, P., \& Boyd, R. (2009). Do Early Intervention Programs Improve Cognitive and Motor Outcomes for Preterm Infants after Discharge? A Systematic Review. Developmental Medicine and Child Neurology, 51, 851-859. https://doi.org/10.1111/j.1469-8749.2009.03414.x

Pekçetin, S., Akı, E., Üstünyurt, Z., \& Kayıhan, H. (2016). The Efficiency of Sensory Integration Interventions in Preterm Infants. Perceptual and Motor Skills, 123, 411-423. https://doi.org/10.1177/0031512516662895

Pipper, M. C., \& Darrah, J. (1994). Motor Assessment of the Developing Infant. Philadelphia, PA: W. B. Saunders Co.

Stecker, P. M., Lembke, E. S., \& Foegen, A. (2008). Using Progress-Monitoring Data to Improve Instructional Decision-Making. Preventing School Failure, 52, 48-58. https://doi.org/10.3200/PSFL.52.2.48-58

Stephens, B. E., \& Vohr, B. R. (2009). Neurodevelopmental Outcome of the Premature Infant. Pediatric Clinics of North America, 56, 631-646.

https://doi.org/10.1016/j.pcl.2009.03.005

Syrengelas, D., Siahanidou, T., Kourlaba, G., Kleisiouni, P., Bakoula, C., \& Chrousos, G. P. (2010). Standartization of the Alberta Infant Motor Scale in Full-Term Greek Infants: Preliminary Results. Early Human Development, 86, 245-249. https://doi.org/10.1016/j.earlhumdev.2010.03.009

Van Hus, J. W. P., Jeukens-Visser, M., Koldewijn, K., Van Sonderen, L., Kok, J. H., Nollet, F., \& Van Wassenaer-Leemhuis, A. G. (2013). Comparing Two Motor Assessments Tools to Evaluate Neurobehavioral Intervention Effects in Infants with Very Low Birth Weight at 1 Year. Physical Therapy, 93, 1475-1483. https://doi.org/10.2522/ptj.20120460

Volpe, J. J. (2009). Brain Injury in Premature Infants: A Complex Amalgam of Destructive and Developmental Disturbances. The Lancet Neurology, 8, 110-124. https://doi.org/10.1016/S1474-4422(08)70294-1

WHO (2012). Born Too Soon: The Global Action Report on Preterm Birth (pp. 8-32). Geneva, Switzerland: World Health Organization. 